\title{
Mean field phase synchronization between chimera states
}

Cite as: Chaos 28, 091101 (2018); https://doi.org/10.1063/1.5049750

Submitted: 25 July 2018 . Accepted: 15 August 2018 . Published Online: 11 September 2018

Ralph G. Andrzejak, Giulia Ruzzene, Irene Malvestio, Kaspar Schindler (D), Eckehard Schöll (iD), and Anna Zakharova
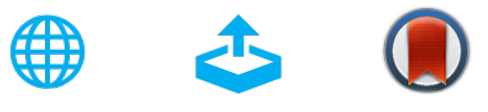

\section{ARTICLES YOU MAY BE INTERESTED IN}

Synchronization of chimera states in a multiplex system of phase oscillators with adaptive couplings

Chaos: An Interdisciplinary Journal of Nonlinear Science 28, 093115 (2018); https:// doi.org/10.1063/1.5031681

\section{Generalized synchronization between chimera states}

Chaos: An Interdisciplinary Journal of Nonlinear Science 27, 053114 (2017); https:// doi.org/10.1063/1.4983841

Existence and stability of chimera states in a minimal system of phase oscillators Chaos: An Interdisciplinary Journal of Nonlinear Science 28, 103121 (2018); https:// doi.org/10.1063/1.5044750

\section{Scilight}

Highlights of the best new research in the physical sciences 


\title{
Mean field phase synchronization between chimera states
}

\author{
Ralph G. Andrzejak, ${ }^{1,2}$ Giulia Ruzzene, ${ }^{1}$ Irene Malvestio, ${ }^{1,3,4}$ Kaspar Schindler, ${ }^{5}$ Eckehard \\ Schöll, ${ }^{6}$ and Anna Zakharova ${ }^{6}$ \\ ${ }^{1}$ Department of Information and Communication Technologies, Universitat Pompeu Fabra, Carrer Roc \\ Boronat 138, 08018 Barcelona, Catalonia, Spain \\ ${ }^{2}$ Institute for Bioengineering of Catalonia (IBEC), The Barcelona Institute of Science and Technology, Baldiri \\ Reixac 10-12, 08028 Barcelona, Spain \\ ${ }^{3}$ Department of Physics and Astronomy, University of Florence, Via Giovanni Sansone 1, 50019 Sesto Fiorentino, \\ Italy \\ ${ }^{4}$ Institute for Complex Systems, CNR, Via Madonna del Piano 10, 50019 Sesto Fiorentino, Italy \\ ${ }^{5}$ Department of Neurology, Sleep-Wake-Epilepsy-Center, Inselspital, University Hospital, University Bern, \\ Freiburgstrasse 18, 3010 Bern, Switzerland \\ ${ }^{6}$ Institut für Theoretische Physik, Technische Universität Berlin, Hardenbergstrasse 36, 10623 Berlin, Germany
}

(Received 25 July 2018; accepted 15 August 2018; published online 11 September 2018)

\begin{abstract}
We study two-layer networks of identical phase oscillators. Each individual layer is a ring network for which a non-local intra-layer coupling leads to the formation of a chimera state. The number of oscillators and their natural frequencies is in general different across the layers. We couple the phases of individual oscillators in one layer to the phase of the mean field of the other layer. This coupling from the mean field to individual oscillators is done in both directions. For a sufficient strength of this inter-layer coupling, the phases of the mean fields lock across the two layers. In contrast, both layers continue to exhibit chimera states with no locking between the phases of individual oscillators across layers, and the two mean field amplitudes remain uncorrelated. Hence, the networks' mean fields show phase synchronization which is analogous to the one between low-dimensional chaotic oscillators. The required coupling strength to achieve this mean field phase synchronization increases with the mismatches in the network sizes and the oscillators' natural frequencies. Published by AIP Publishing. https://doi.org/10.1063/1.5049750
\end{abstract}

Chimera states are an intriguing coexistence of synchronous and asynchronous motion in networks of coupled dynamics. ${ }^{1,2}$ There is growing evidence that the analysis of chimera states can help understand the dynamics of natural and man-made networks. ${ }^{3-5}$ In such realworld dynamics, however, the interplay of synchronization and desynchronization is not only important within individual networks but also across interacting networks. ${ }^{6}$ Therefore, while chimera states were traditionally studied in one-layer networks, recent work deals with interactions of chimera states across coupled layers in multilayer networks. ${ }^{7-19}$ In particular, it was shown in Ref. 12 that driver-response couplings between layers can induce socalled generalized synchronization, ${ }^{20-22}$ where the state of the response layer becomes a unique function of the state of the driver layer. While these findings on generalized synchronization between chimera states ${ }^{12}$ are appealing from a theoretical point of view, their applicability might remain limited because this particular type of synchronization is difficult to detect. Moreover, previous work ${ }^{7-17}$ focused on multiplex networks, for which the layers must have the same number of oscillators and the inter-layer coupling connects individual oscillators in a pairwise and ordered topology. These constraints exclude many types of real-world multilayer networks, which in general can have different numbers of nodes in each layer, thereby already ruling out a multiplex connection topology across layers. Furthermore, the nodes in the network of one layer might not be directly connected to the nodes in the network of the other layer but instead to some macroscopic variable of the other network. We, therefore, use a more general approach than in previous work $^{7-19}$ and study two-layer networks with a different number of oscillators in each layer. For each layer, we use the classical setting of a ring network of non-locally coupled identical phase oscillators in a chimera state. We at first briefly illustrate that chimera states in individual ring networks can be identified as weakly chaotic attractors (Refs. 5, 23, and 24 and references therein). We then couple the phases of individual oscillators in one layer to the phase of the mean field of the other layer. This coupling from a macroscopic variable in one layer to the microscopic variables in the other layer is done in both directions. We show that also in the presence of inter-layer coupling, both layers continue to exhibit chimera states. For a sufficient coupling strength, the phases of the two mean fields lock, while their amplitudes remain uncorrelated. Hence, in analogy to low-dimensional chaotic oscillators, ${ }^{25}$ the mean fields of networks in chimera states can show phase synchronization. We then study the parameter plane spanned by the mismatch in the natural frequencies of the two layers and the coupling strength between them. We show that the synchronization region in this plane forms Arnold tongues, ${ }^{26}$ a finding which further strengthens the analogy to the low-dimensional case dealt with in Ref. 25 . We discuss the applicability of our findings using examples from neuroscience. In particular, we propose that our study may shed light on the dynamics and action of weak electromagnetic fields emerging from the collective activity of biological neuronal networks. 


\section{INTRODUCTION}

Interacting networks are ubiquitous in nature. In many cases, such as brain networks, neither a completely synchronized nor a completely desynchronized dynamics of the networks would allow for a proper function. Instead, a balance between synchronization and desynchronization is essential not only within networks but also across networks. Recent work on chimera states, which are paradigmatic for this balance, has, therefore, progressed from the study of individual networks to interacting networks. Chimeras were studied in multilayer networks of phase oscillators, $7,11-13,16,19$ Hindmarsh-Rose, ${ }^{7,9,10,14,18}$ and FitzHugh-Nagumo ${ }^{27}$ model neurons, as well as chaotic time-discrete maps. ${ }^{8,15,17}$ It was shown that couplings between network layers can suppress or induce ${ }^{7-11,14-17}$ chimera states in individual layers and that chimeras can be identical, almost identical, or distinct across different layers. ${ }^{7,8,11-16,18}$ Coupling delays $^{8-10}$ as well as parameter mismatches or structural differences across layers ${ }^{7,9,11,15-17}$ were found to play an important role in this multilayer setting. For the special case of bipartite networks in-phase, anti-phase and out-of-phase chimeras were observed. ${ }^{19,27}$

To address the specific issue of across-layer synchronization, we previously studied a two-layer multiplex network with a driver-response coupling scheme. ${ }^{12}$ Each layer consisted of a ring network of $N$ non-locally coupled identical phase oscillators, ${ }^{1,2}$ and both layers individually showed chimera states when there was no connection between them. We then applied a unidirectional coupling from the driver layer $X$ to the response layer $Y$. Each node in the layer $X$ with index $j$ was connected to its counterpart node in $Y$ with index $j$, for $j=1 \ldots N$. For a sufficiently strong coupling, the layers entered into generalized synchronization. This particular kind of synchronization is defined by the existence of a function $H$ that determines the state of the response, given the state of the driver: $Y=H(X) .{ }^{20-22}$ Despite this synchronization across layers, both networks continued to show chimera states with distinct spatiotemporal dynamics. Since synchronization can play a role in the transmission of information across interacting networks in nature, we concluded in Ref. 12 that our findings on generalized synchronization led to a broader applicability of the concept of chimera states to real-world phenomena. It can be objected, however, that the function $H$ can be very complicated, and a direct test for its existence based on the observation of the temporal evolution of $X$ and $Y$ requires the computation of conditional Lyapunov exponents (Refs. 26 and 28 and references therein) or some advanced nonlinear signal analysis measures (e.g., Ref. 29). For our model systems studied in Ref. 12, we detected generalized synchronization indirectly using the auxiliary system approach. ${ }^{21,22}$ For this approach, however, one has to construct an exact replica of the response network, and it is unlikely that such a technique can be directly applied in real-world systems. Furthermore, for the multiplex setting used in Refs. 7-18, the layers must have the same number of nodes $N$, and connections between nodes across the layers are pairwise and ordered. As stated above, these constraints exclude many types of networks. Finally, inter-layer connections in multilayer networks can be bidirectional, and the work of Ref. 12 was restricted to unidirectional driverresponse couplings. In the present work, we overcome these limitations.

\section{TWO-LAYER NETWORK}

We analyze a two-layer network and denote the individual layers by $X$ and $Y$. Each layer is constituted by a ring network of $N_{x}$ and $N_{y}$ phase oscillators, respectively. Within each layer the phase oscillators are mutually coupled via a non-local rectangular kernel. As a consequence, the networks of each layer enter into a chimera state. We denote the timedependent phases of individual oscillators in $X$ by $\phi_{x, j_{x}}(t)$ for $j_{x}=1, \ldots, N_{x}$, and their mean field is quantified by the complex Kuramoto order parameter ${ }^{30}$

$$
Z_{x}(t)=\frac{1}{N_{x}} \sum_{j_{x}=1}^{N_{x}} e^{i \phi_{x, j}(t)}=R_{x}(t) e^{i \Phi_{x}(t)},
$$

with collective amplitude $R_{x}(t)$ and phase $\Phi_{x}(t)$. This order parameter rotates in the complex plane, and its phase velocity is denoted by $\Omega_{x}(t)=d \Phi_{x}(t) / d t$. All quantities are defined analogously for $Y$.

To connect the two layers, the phases of all oscillators in $X$ are coupled with strength $\varepsilon$ to the order parameter phase of the network $Y$. In the same way, the phases of all oscillators in $Y$ are coupled with the same strength $\varepsilon$ to the order parameter phase of the network $X .{ }^{31}$ Overall, this leads to

$$
\begin{aligned}
\dot{\phi}_{x, j_{x}}(t)= & \omega_{x}-\varepsilon \sin \left[\phi_{x, j_{x}}(t)-\Phi_{y}(t)\right] \\
& -\frac{1}{2 b_{x}} \sum_{k=j_{x}-b_{x}}^{j_{x}+b_{x}} \sin \left[\phi_{x, j_{x}}(t)-\phi_{x, k}(t)+\alpha\right]
\end{aligned}
$$

and analogously for $Y$. The phase lag parameter is set to $\alpha=1.46 .^{12,23,24,32}$ The number of oscillators $N_{x, y}$ is in general different for $X$ and $Y$. The natural frequencies of individual oscillators $\omega_{x, y}$ are the same for all oscillators within a given layer, but in general different across the layers $X$ and $Y$. The coupling range is set to $b_{x, y}=0.35 \cdot N_{x, y}$. According to the networks' ring architecture, sums and differences of oscillator indices in Eq. (2) are to be understood as modulo $N_{x, y}$.

\section{INTEGRATION OF DYNAMICS AND PRELIMINARIES}

The networks of both layers $X$ and $Y$ were initialized with random phases independently and uniformly distributed in $(0,2 \pi]$, and we integrated the dynamics using a fourth-order Runge-Kutta scheme with fixed sampling time of $d t=0.05$. Results of pre-analysis comparing $d t=0.01$ and $d t=0.05$ showed the latter to lead to a sufficient accuracy. For each run, we integrated the dynamics for a total of $2 \cdot 10^{6}$ sampling times, corresponding to $1 \cdot 10^{5}$ dimensionless time units. At the parameters we used, the complex order parameters revolve on the order $1.25 \cdot 10^{4}$ times in this period. The coupling between layers $\varepsilon$ was turned on only after the first $1 \cdot 10^{3}$ time units, so that initially the networks in both layers could settle to a chimera state not being influenced by the other layer. The resulting dynamics was evaluated during the interval $I=\left[1 \cdot 10^{4}, 1 \cdot 10^{5}\right]$ time units. Accordingly, transients 


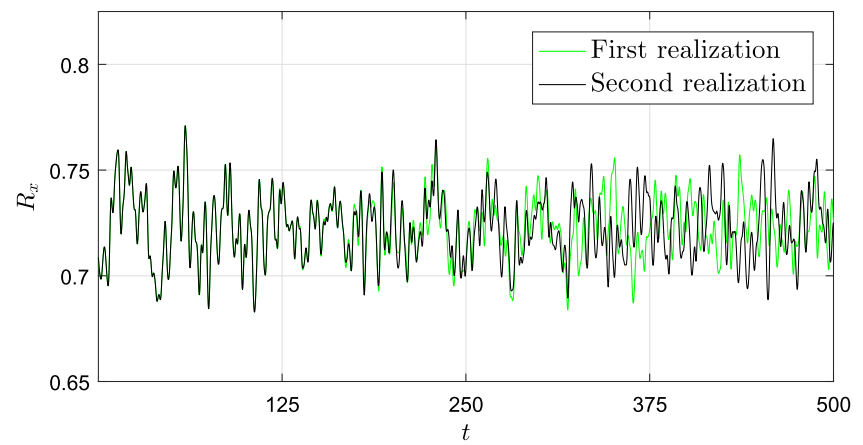

FIG. 1. The mean field of a network in a chimera state is sensitively dependent on the initial conditions. Temporal evolution of the order parameter amplitude $R_{x}(t)$ obtained for two almost identical initial conditions in a chimera state.

toward a synchronized motion could fade out between $1 \cdot 10^{3}$ and $1 \cdot 10^{4}$ time units.

For our finite-size ring networks, chimera states can suddenly collapse to a fully coherent state ${ }^{24}$ in which the phases of all nodes become locked and oscillate at constant phase velocity. Furthermore, after the initialization with random phases the network can directly go to this fully coherent state without ever forming a chimera state. While chimera state collapses were studied in Refs. 24 and 32, we follow Ref. 12 and exclude them from the present study. Whenever the network of either of the layers approached the fully coherent state, the realization was discarded and a new realization was started with new random initial conditions. This had to be done only occasionally, since at the network sizes we used the mean lifetime of chimera states is orders of magnitudes higher than the evaluation interval $I{ }^{24,32}$ It was, therefore, not necessary to further increase the stability of the chimera states by using a higher number of oscillators.

Chimera states in finite-size networks can be identified as weakly chaotic attractors (Refs. 5, 23, and 24 and references therein). Accordingly, the mean field of a network in a chimera state can be regarded as a chaotic oscillator. To illustrate this fact we started the network of layer $X$ twice with almost identical initial conditions for $N_{x}=250, \omega_{x}=0$, and $\varepsilon=0$, i.e., uncoupled from the network of layer $Y$. Both initial conditions were already in a chimera state and differed only by 0.01 in the phase of one single oscillator from the unsynchronized group. As a consequence of this difference, the order parameters of both realizations diverged rapidly and got completely uncorrelated. This sensitive dependence on the initial conditions is illustrated in Fig. 1 using the order parameter amplitude (see also Fig. 2 of Ref. 23). This illustration serves merely as an example for the well-established chaotic nature of chimera states, and we include it only to underline that our results indeed concern the synchronization of chaotic oscillators.

\section{QUANTIFICATION OF PHASE SYNCHRONIZATION}

To assess the synchronization between the mean fields of the two layers $X$ and $Y$, we analyze their rotating order parameters $Z_{x}(t)$ and $Z_{y}(t) .{ }^{33}$ At first, we define

$$
\Delta \Phi(t)=\Phi_{x}(t)-\Phi_{y}(t) .
$$

Phase synchronization of the order parameters can be defined using the strong locking condition $\Delta \Phi(t)=$ const or the weak locking condition $|\Delta \Phi(t)|<$ const. $^{25}$ To distinguish the regimes of strong phase locking, weak phase locking, and unsynchronized motion, we introduce the normalized phase difference index:

$$
S=\frac{\max \{\Delta \Phi(t)\}-\min \{\Delta \Phi(t)\}}{2 \pi} .
$$

Here, curly brackets denote the set of values across all times included in the evaluation interval, i.e., $t \in I$. The different regimes can be distinguished as follows. Phase synchronization detected from strong phase locking, $S=0$ : If $\Phi_{x}(t)$ and $\Phi_{y}(t)$ rotate with a constant difference, we get $\max \{\Delta \Phi(t)\}=\min \{\Delta \Phi(t)\}$ and accordingly $S=0$. This includes $\Delta \Phi=$ const $>0$ which corresponds to phase synchronization with a constant phase lag. Phase synchronization detected from weak phase locking, $0<S<1$ : Any temporal variability of $\Delta \Phi(t)$ leads to $S>0$. However, as long as the order parameters never lap each other in the complex plane, i.e., the difference of their phases stays below $2 \pi, S$ remains below 1 . The smaller the temporal variability of $\Delta \Phi(t)$, the smaller the value of $S$. Unsynchronized motion, $S \geq 1$ : As soon as one order parameter completes at least one more rotation than the other order parameter, $\Delta \Phi(t)$ grows by $2 \pi$ and we get $S \geq 1$. This indicates that the phases are not locked. The order parameter do not rotate in a phase synchronized manner.

Inter-layer couplings $\varepsilon$ that are not strong enough to lead to a phase synchronized motion of the mean fields can still induce a dependence between their phases. We quantify the degree of this dependence by the temporal mean phase difference coherence:

$$
C=\left|\left\langle\left\{e^{i \Delta \Phi(t)}\right\}\right\rangle\right|
$$

Here, angular brackets \langle\rangle denote the temporal average taken over the values included in the set defined by \{\} and $\|$ denotes the absolute value. Accordingly, $C$ can also be read as the modulus of the complex Kuramoto order parameter of the phase differences $\Delta \Phi(t)$. If the two order parameters evolve independently, $\Delta \Phi(t)$ is uniformly distributed in $(0,2 \pi]$, and we get $C=0 .{ }^{34}$ If, due to a coupling, $\Delta \Phi(t)$ is attracted by some phase lag, it is no longer uniformly distributed across the circle. Instead, the distribution of $\{\Delta \Phi(t)\}$ is peaked around this attracting value, and we get $C>0$. Once the strong phase locking condition is fulfilled $C$ reaches its upper bound of 1 . However, in contrast to $0<S<1$, the measure $C$ has no defined range if the weak phase locking condition is fulfilled. The advantage of $C$ over $S$ is that $C>0$ allows us to detect dependencies in the phases in the unsynchronized regime even if $\Delta \Phi(t)$ suffers frequent slips by $2 \pi$ resulting in $S \gg 1$.

Finally, the Pearson correlation coefficient ${ }^{35}$ between the order parameter amplitudes taken across the evaluation interval $I$ is denoted by $K$. If these amplitudes $\left\{R_{x}(t)\right\}$ and $\left\{R_{y}(t)\right\}$ are independent variables, the expected value is $K=0$. The upper limit of $K=1$ would be obtained for identical synchronization of the amplitudes $R_{x}(t)=R_{y}(t)$. 


\section{RESULTS}

We start by inspecting the dynamics with no coupling between the layers, that is, Eq. (2) with $\varepsilon=0$. The other parameters are set to $N_{x}=250, N_{y}=150, \omega_{x}=0.01, \omega_{y}=0$ and the fixed values specified in Sec. II for the remaining quantities (Fig. 2). As a consequence of the non-local coupling within the networks of individual layers, they are both in chimera states [Fig. 2(a)]. On the other hand, because of the lack of inter-layer coupling, the two networks evolve independently from another. The difference between the order parameter phases $\Delta \Phi(t)$ increases linearly with some superimposed fluctuations [Fig. 2(b)]. These fluctuations are caused by the diffusion of the individual mean field phases, ${ }^{26}$ reflecting the chaotic nature of the chimera dynamics (see again Fig. 1). The overall increase of $\Delta \Phi(t)$ shows that the layers' complex mean fields rotate at different mean phase velocities $\Omega_{x}(t)$ and $\Omega_{y}(t)$. This difference is caused by the mismatches in the number of oscillators, $N_{x}$ versus $N y$, and in the natural frequencies of individual oscillators, $\omega_{x}$ versus $\omega_{y}$. The amplitudes of the order parameters $R_{x}(t)$ and $R_{y}(t)$ show temporal fluctuations, and there is no relation between them [Fig. 2(c)]. When taken for the entire evaluation interval $I$, the Pearson correlation coefficient between $R_{x}(t)$ and $R_{y}(t)$ is $K=0.006$.

When the networks are mutually coupled with $\varepsilon=0.01$, both networks continue to exhibit chimera states [Fig. 3(a)]. In contrast to the uncoupled case, however, the order parameter phase difference $\Delta \Phi(t)$ remains bounded; it fluctuates around a mean value of approximately $0.16 \pi$ [Fig. 3(b)]. This indicates that while the strong phase locking condition is not met, the weak phase locking condition is fulfilled. In contrast to the order parameter phases, their amplitudes $R_{x}(t)$ and $R_{y}(t)$ remain uncorrelated over time [Fig. 3(c)], reflected in a negligible value of $K=-0.016$. Accordingly, only the phases but not the amplitudes of the order parameters $Z_{x}(t)$ and $Z_{y}(t)$ are synchronized. Hence, the mean fields of the two layers exhibit a phase synchronized dynamics, analogous to the one described for low-dimensional chaotic oscillators by Rosenblum et al. ${ }^{25}$

In contrast to the phases of the macroscopic mean fields, phases of pairs of individual oscillators are not locked across layers. First of all, as opposed to multiplex networks, in our setting there is no pre-defined pairing between individual oscillators with the same index in both networks. Considering pairings of oscillators with in general different indices, we find that individual pairs are only locked temporarily while both oscillators belong to the group of coherent oscillators in their respective network [Fig. 3(a)]. However, since these coherent groups drift across the networks of the layers, ${ }^{23}$ in the long run, all individual oscillators switch back and forth between their coherent and incoherent groups. As soon as one oscillator is in the incoherent group, the phase locking is broken. Accordingly, there is no stable phase locking between any pair of individual oscillators across the layers.

To further illustrate the transition to mean field phase synchronization, we inspect the temporal evolution of the order parameter phase difference $\Delta \Phi(t)$ for exemplary inter-layer coupling strengths $\varepsilon$ [Fig. 4 and again Figs. 2(b) and 3(b)]. For zero coupling, $\Delta \Phi(t)$ increases in an approximately, though not strictly linear way versus time. For nonzero but nonsynchronizing couplings we find the typical stick-jump cycles (see also Fig. 1 in Ref. 25). In each cycle, $\Delta \Phi(t)$ at first
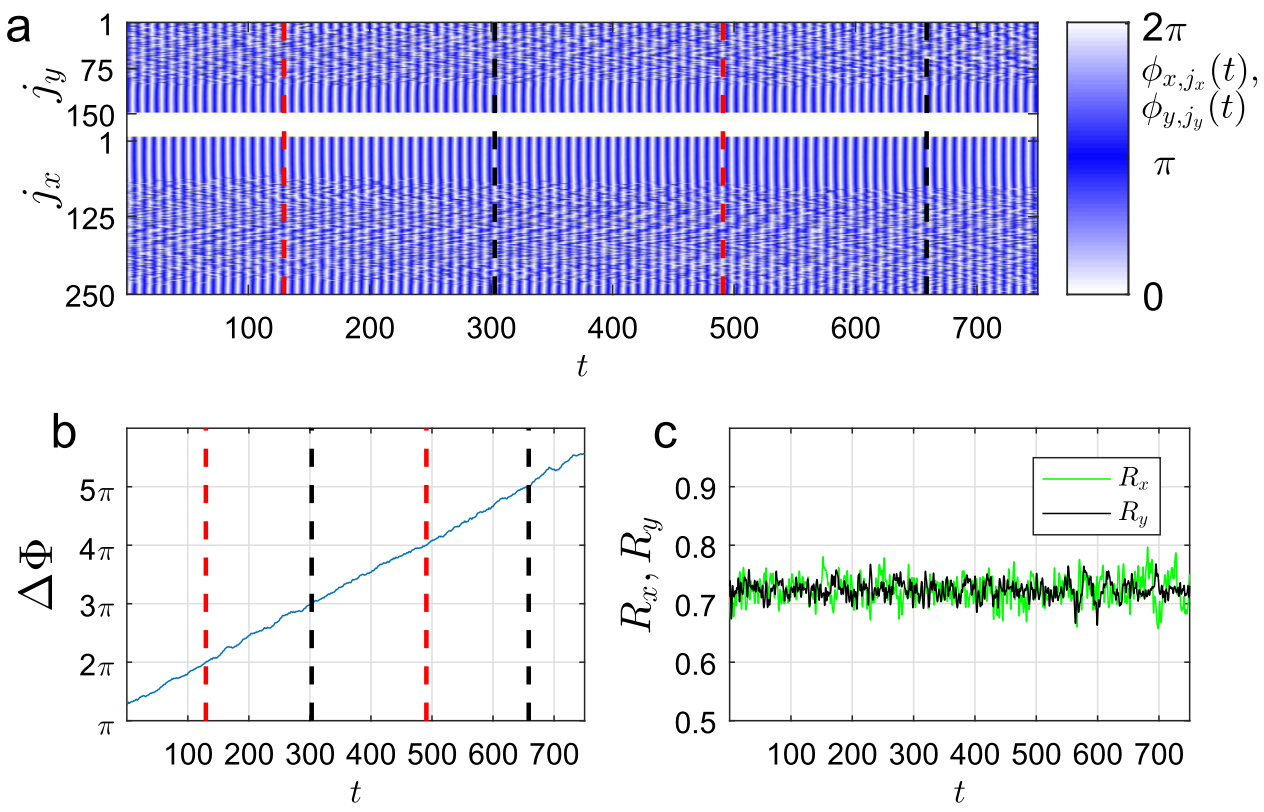

FIG. 2. Without coupling between the layers $X$ and $Y$, the difference between their mean field phases is unbounded. All panels have the same interval of time $t$ as abscissa. Time is relative to the beginning of the evaluation interval $I$, which has a total duration of 90000 time units. We use $\omega_{x}=0.01, \omega_{y}=0, N_{x}=250$, $N_{y}=150$, and accordingly $b_{x}=87, b_{y}=52$. Panel (a) shows the temporal evolution of phases of the individual oscillators $\phi_{x, j_{x}}(t)$ and $\phi_{y, j_{y}}(t)$ obtained from an exemplary solution of Eq. (2) for $\varepsilon=0$. The networks of the layers $X$ and $Y$ are displayed at bottom and top, respectively, separated by the white gap. For each layer separately, we shifted the oscillator indices circularly such that the groups of coherent oscillators of $X$ and $Y$ are adjacent in this display. This can be done due to the ring architecture of the networks. Panel (b) shows the order parameter phase difference $\Delta \Phi(t)$. In both panels (a) and (b), the red and black vertical lines indicate the instants when the order parameters are in-phase and in anti-phase, respectively. Panel (c) shows the evolution of the order parameter amplitudes $R_{x}(t)$ and $R_{y}(t)$. 

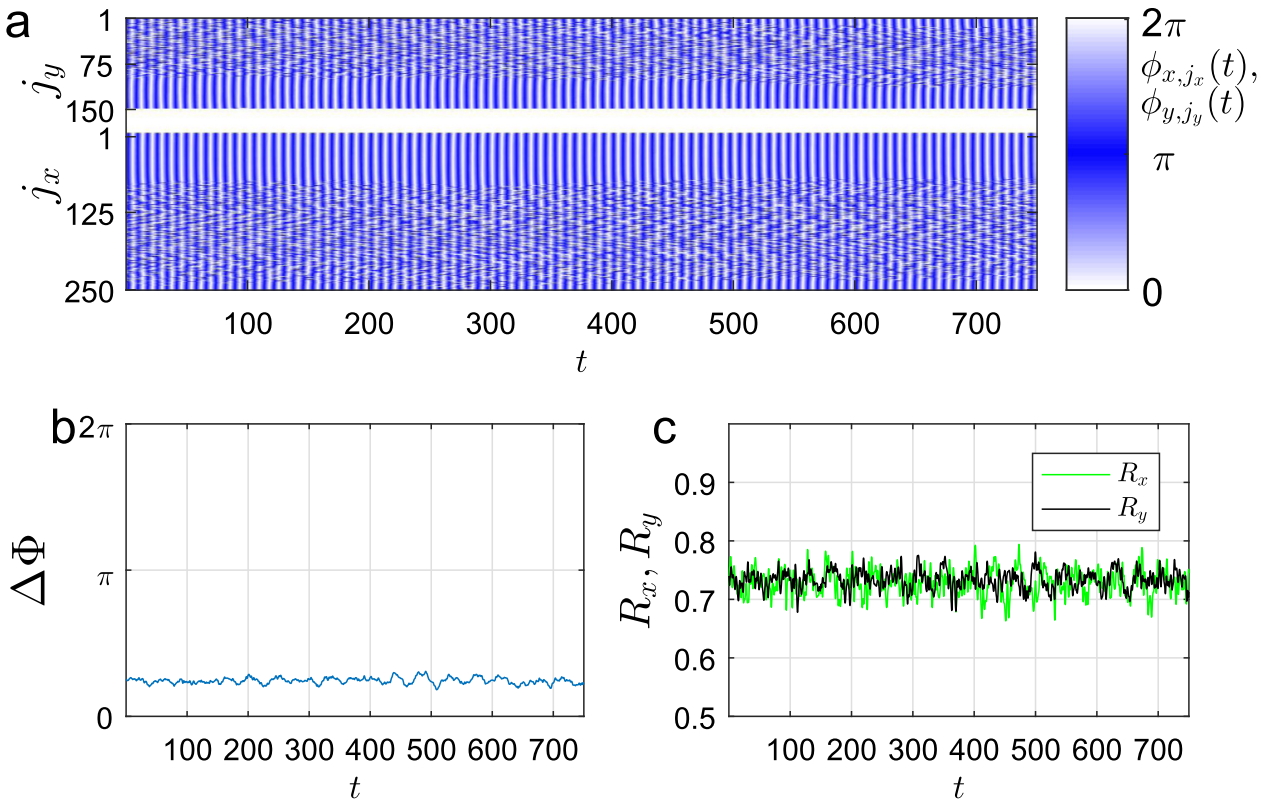

FIG. 3. For sufficiently strong coupling between the networks of the layers $X$ and $Y$, their mean fields are phase synchronized. Same as Fig. 2 but for $\varepsilon=$ 0.01 . No red or black vertical lines are used since the order parameters are never in-phase or in anti-phase. increases very slowly (stick) and then rapidly completes a full rotation of $2 \pi$ (jump). The duration of these cycles fluctuates but generally increases with the coupling strength. Finally, for sufficient coupling, $\Delta \Phi(t)$ remains bounded and does not complete rotations of $2 \pi$ any longer.

Figure 5 displays results obtained upon a gradual increase of the coupling strength between layers $\varepsilon$. For $\varepsilon=0$, the unbounded growth of $\Delta \Phi(t)$ results in $S \gg 1$ [Fig. 5(a)]. This normalized phase difference index $S$ remains limited only due to the finite duration of the evaluation interval $I$. Furthermore, at $\varepsilon=0$, the temporal mean phase difference coherence $C$ is indistinguishable from zero, reflecting that

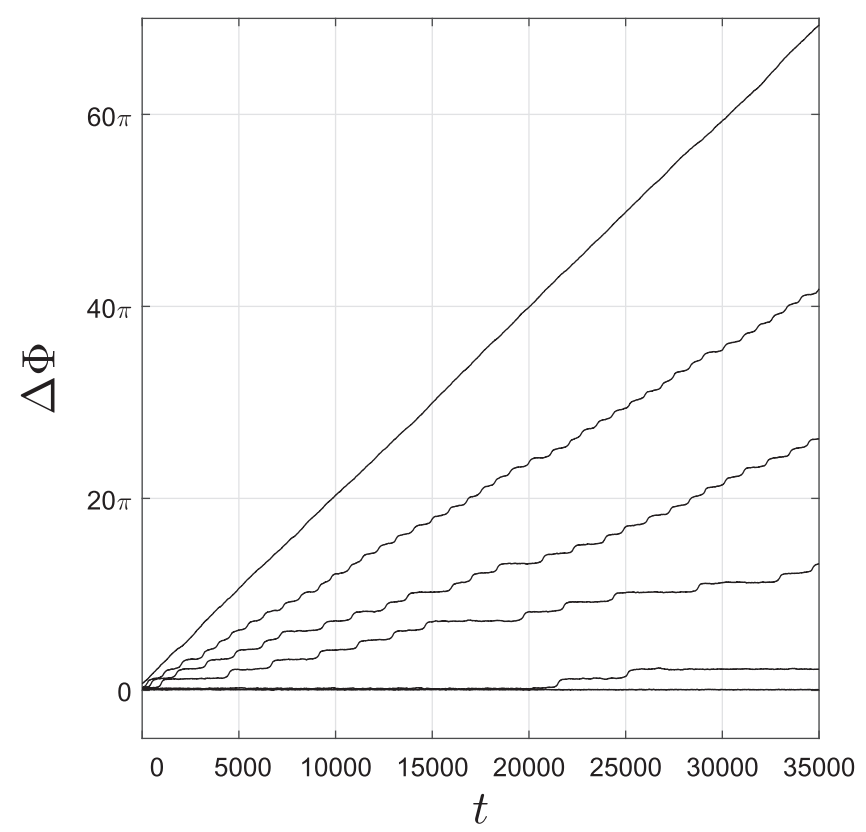

FIG. 4. Increasing the inter-layer coupling strength prolongs the periods in which the phase difference is stuck. Temporal evolution of the order parameter phase difference $\Delta \Phi(t)$ for different coupling strengths between layers: $\varepsilon=0 ; 0.004 ; 0.0045 ; 0.004875 ; 0.005125 ; 0.01$ from top to bottom at the right border of the plot. All other parameters are like in Figs. 2 and 3.
$\Delta \Phi(t)$ is uniformly distributed across the circle [Fig. 5(b)]. Since the order parameters rotate clockwise, their mean phase velocities are negative. For $\varepsilon=0$, we find that $\left|\left\langle\left\{\Omega_{y}(t)\right\}\right\rangle\right|>$ $\left|\left\langle\left\{\Omega_{x}(t)\right\}\right\rangle\right|$, i.e., the order parameter $Z_{y}(t)$ rotates faster than $Z_{x}(t)$ [Fig. 5(c)]. For nonzero but low couplings we still get $S \gg 1$. This shows that $\Delta \Phi(t)$ continues to undergo frequent jumps by $2 \pi$, each incrementing $S$ by one. Nonetheless, when taken modulo $2 \pi, \Delta \Phi(t)$ is already attracted by a certain phase difference resulting in $C>0$ (see again Fig. 4). Around $\varepsilon \approx 0.005$, the measure $S$ drops sharply, has first entries below 1 for $\varepsilon>\varepsilon_{1}=0.0053125$, and remains below 1 for $\varepsilon>\varepsilon_{2}=0.005475$. In $\varepsilon_{1}<\varepsilon<\varepsilon_{2}$, we find some realizations that perform a $2 \pi$ phase difference jump during the evaluation interval $(S>1)$ and some realizations that do not $(S<1)$. Such behavior is common in synchronization transitions; see, for instance, the synchronization transition found in delay-coupled FitzHugh-Nagumo systems with excitatory and inhibitory links (Fig. 3 in Ref. 36). It indicates that the synchronization transition in networks is a non-equilibrium phase transition of second order, not of first order, i.e., it is not a discontinuous transition in systems with finite $\mathrm{N}$. Above $\varepsilon_{2}$ we find that $\left\langle\left\{\Omega_{x}(t)\right\}\right\rangle$ is very close to $\left\langle\left\{\Omega_{y}(t)\right\}\right\rangle$, and $C$ approaches its upper bound of 1 , though without reaching it. Most importantly, we find $0<S<1$. Hence, the weak phase locking, but not the strong phase locking condition, is fulfilled. Throughout the range of the coupling, the Pearson correlation coefficient $K$ between the order parameter amplitudes remains vanishingly small [Fig. 5(d)]. Overall, Fig. 5 shows that for $\varepsilon>\varepsilon_{2}$, the mean fields of the two layers are phase synchronized.

We now consider the parameter plane spanned by $\omega_{x}$ and $\varepsilon$, while we continue to keep $N_{x}=250, N_{y}=150, \omega_{y}=0$ fixed [Figs. 6(a) and 6(b)]. Throughout the range of $\omega_{x}$ and $\varepsilon$, the correlation between the order parameter amplitudes remained $-0.03<K<0.04$ (not shown). Already at $\omega_{x}=$ $\omega_{y}=0$, a substantial coupling is needed to achieve the weak phase locking condition [Fig. 6(a)]. The reason is that the difference in the number of oscillators $N_{x}$ and $N_{y}$ by itself 
a

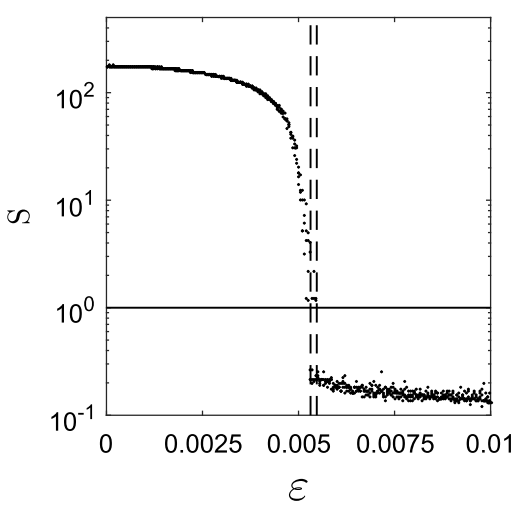

C

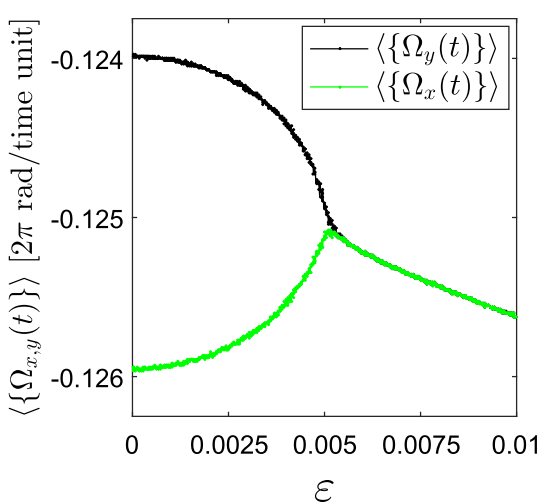

b

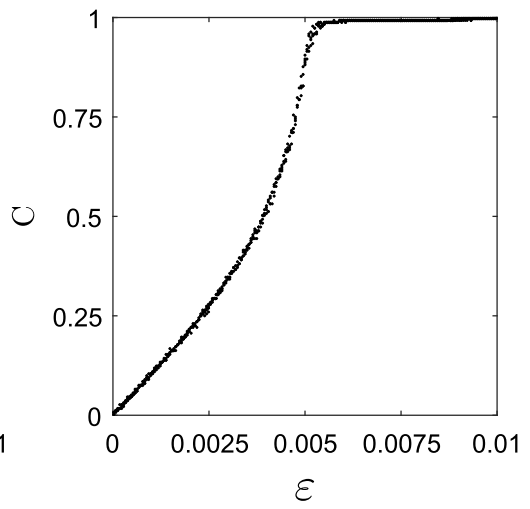

d

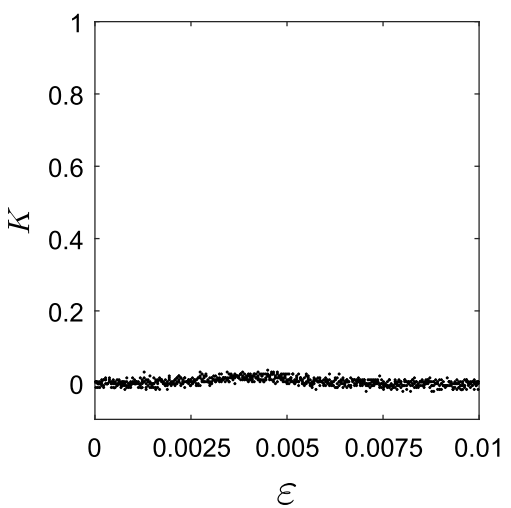

FIG. 5. Transition to the phase synchronized motion of the mean fields upon increasing the coupling between layers. All panels have the same interval of the coupling strength between layers $\varepsilon$ as abscissa. All other parameters are like in Figs. 2 and 4. (a) Normalized phase difference index $S$. For realizations below the horizontal black line at $S=$ 1 , the weak phase locking condition is fulfilled. The vertical dashed lines indicate from left to right $\varepsilon_{1}$ and $\varepsilon_{2}$. (b) Temporal mean phase difference coherence $C$. (c) Mean order parameter phase velocities $\left\langle\left\{\Omega_{x}(t)\right\}\right\rangle,\left\langle\left\{\Omega_{y}(t)\right\}\right\rangle$. (d) The Pearson correlation coefficient $K$ between the order parameter amplitudes. leads to a difference in $\Omega_{x}(t)$ and $\Omega_{y}(t)$. Even if $\omega_{x}=\omega_{y}=0$, the order parameter $Z_{y}(t)$ rotates faster than $Z_{x}(t)$, and interlayer coupling is needed to overcome this mismatch in the phase velocities of the mean fields. Increasing the natural frequency $\omega_{x}$ of individual oscillators in the layer $X$ while keeping $\omega_{y}=0$, increases this mismatch further. As a consequence, higher coupling strengths are needed to achieve phase synchronization between the mean fields. The required coupling strength increases approximately linearly with $\omega_{x}$. Note that $\omega_{x}$ directly corresponds to the difference in the oscillators' natural frequencies since we keep $\omega_{y}=0$. Furthermore, we find that synchronization is established with a non-zero lag between $\Phi_{x}$ and $\Phi_{y}$, and this lag is reduced upon increasing $\varepsilon$ [Fig. 6(b)]. a
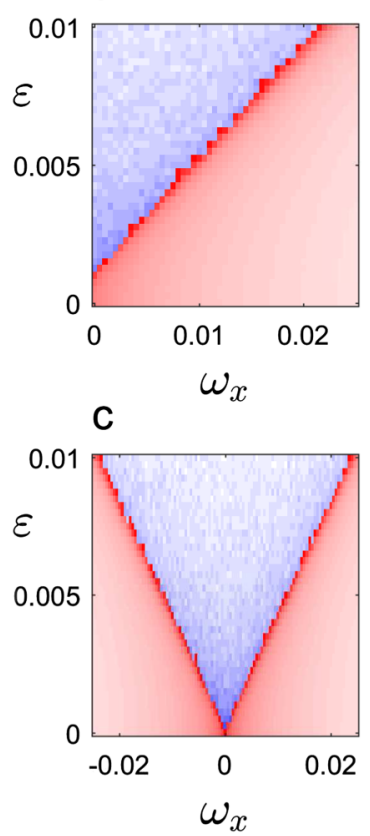

b
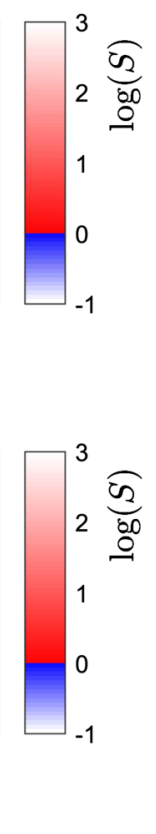

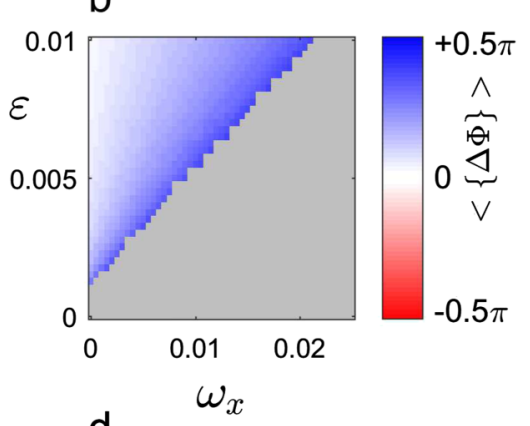

d

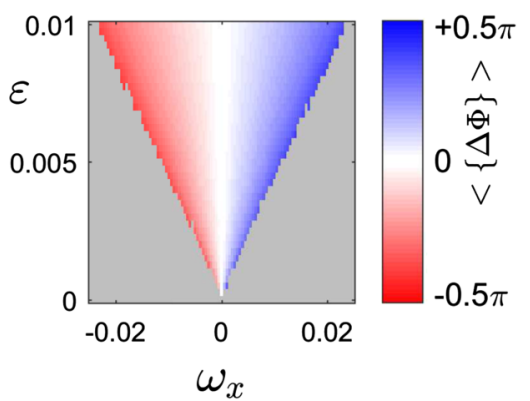

FIG. 6. Stronger couplings are needed to overcome frequency mismatches and reduce phase lags in synchronized motion. Dependence of results on the coupling strength between layers $\varepsilon$ and the natural frequency $\omega_{x}$ of oscillators in layer $X$. We fix $\omega_{y}=0$. [Panel (a)] Logarithm of the normalized phase difference index $S$ for $N_{x}=250, N_{y}=$ 150 , and accordingly $b_{x}=87, b_{y}=52$. Blue colors indicate that the weak phase locking condition $(0<S<1)$ is fulfilled. Red colors indicate that the mean field phases remain unlocked. [Panel (b)] Mean phase difference $\langle\{\Delta \Phi(t)\}\rangle$ taken modulo $2 \pi$. Values are displayed only for combinations of $\varepsilon$ and $\omega_{x}$ for which $S<1$ in panel (a). The remaining cases are shown in grey. Panels (c) and (d) same as (a) and (b) but for $N_{x}=N_{y}=200$ and $b_{x}=b_{y}=70$. 
As last setting, we study the case when both layers have the same number of oscillators $N_{x}=N_{y}=200$ [Figs. 6(c) and 6(d)]. We find that for $\omega_{x} \rightarrow \omega_{y}$, synchronization already occurs for very small couplings [Fig. 6(c)]. This was also reported for the chaotic but highly coherent Rössler dynamics in Ref. 25. It implies that the diffusion of the order parameter phases is only moderate. A further analogy to the lowdimensional case first studied by Rosenblum and colleagues is that the synchronization regions shown in Fig. 6 form Arnold tongues, i.e., the phase-locking domain for coupled periodic oscillators (see Fig. 2 in Ref. 25). For $\omega_{x}=\omega_{y}=0$, the mean phase lag $\langle\{\Delta \Phi\}\rangle$ becomes very close to zero [Fig. 6(d)]. Importantly, also for this zero-lag phase synchronization of the mean fields, their amplitudes $R_{x}(t)$ and $R_{y}(t)$ remain uncorrelated over time. The correlation coefficient between these amplitudes was found to be $-0.034<K<0.042$ across all phase synchronized solutions (not shown). In summary of our last setting, also if the networks constituting the two layers have the same number of oscillators with the same natural frequency, only the weak phase locking criterion is fulfilled. Like for all results shown above, the strong phase locking condition is not fulfilled. This is to be expected given the chaotic nature of the chimera states for our finite size networks. . $^{53,24}$

\section{DISCUSSION}

Our work provides a conceptual extension of phase synchronization of chaotic oscillators ${ }^{25}$ to networks showing chimera states. Compared to previous work on generalized synchronization of networks in chimera states, ${ }^{12}$ the present work on phase synchronization is of significantly wider applicability. First of all, the multiplex setting with a driverresponse coupling studied in Ref. 12 excludes many types of real-world multilayer networks. Furthermore, to detect generalized synchronization from the temporal evolution of networks requires the computation of conditional Lyapunov exponents $^{26,28}$ or nonlinear interdependence measures. ${ }^{29}$ As an alternative one can use the auxiliary system approach. ${ }^{21,22}$ This seems, however, even more prohibitive in real-world scenarios since an exact replica of the driven network is needed. In contrast, to detect phase synchronization as described in the present work, one only has to monitor whether the difference of the mean field phases across networks remains bounded over time.

Our results are also interesting from a neuroscientific point of view, because they may provide a theoretical framework for modern concepts about how communication is implemented in brain networks. ${ }^{37}$ One important characteristic of brain networks is that most connections are local, probably mostly as a result of the strong evolutionary constraint to minimize energy consumption of long-range axonal transmission. The prevalence of local connections also elegantly explains the well-known $\sim 1 / f$ characteristic of the electroencephalogram (EEG). ${ }^{38}$ However, the activities between different brain regions have to be coordinated, ideally in an energy-efficient way and with minimal delay. ${ }^{39}$ One intriguing possibility of efficient long-range communication are weak endogenous electric fields generated by the (partial) local synchronization of neurons. ${ }^{40}$ In the framework developed in the present study, these emerging electric fields correspond to the mean field order parameters. Spatial orientation of neurons, their morphology, and the distribution of ion channels in their membranes may render sets of neurons specifically responsive to differently oriented electric fields and thus provide the substrate for selective even directed communication as modeled here. ${ }^{41,42}$ If neurons are conceptualized as oscillators, then the sudden change of natural frequencies in a local network as, for example, observed at the onset of epileptic seizures ${ }^{43,44}$ might then also disrupt the coordination conveyed by the phase synchronization of the order parameter and allow pathologic activity to develop and spread, until coupling is increased, for example, by the development of stronger electric fields toward the end of epileptic seizures. ${ }^{45}$ Finally, we propose that the hypothesis of phase synchronization between endogenously generated electric fields as a coordinating mechanism between distant brain regions might be tested by multi-scale electrophysiologic recordings, such as combining single unit, local field potential, and EEG recordings as has become technically feasible in recent years. $^{46}$

\section{ACKNOWLEDGMENTS}

The authors are grateful to Flavio Fröhlich and Ernest Montbrió for useful discussion. We acknowledge funding from the Spanish Ministry of Economy and Competitiveness, Grant No. FIS2014-54177-R (R.G.A and G.R.), the CERCA Programme of the Generalitat de Catalunya (R.G.A), the European Union's Horizon 2020 research and innovation programme under the Marie Sklodowska-Curie Grant Agreement No. 642563 (R.G.A and I.M.), and the Deutsche Forschungsgemeinschaft (DFG) in the framework of SFB 910 (E.S., A.Z., and G.R.).

${ }^{1}$ Y. Kuramoto and D. Battogtokh, "Coexistence of coherence and incoherence in nonlocally coupled phase oscillators," Nonlinear Phenom. Complex Syst. 5, 380-385 (2002).

${ }^{2}$ D. M. Abrams and S. H. Strogatz, "Chimera states for coupled oscillators," Phys. Rev. Lett. 93, 174102 (2004).

${ }^{3}$ M. J. Panaggio and D. M. Abrams, "Chimera states: Coexistence of coherence and incoherence in networks of coupled oscillators," Nonlinearity $\mathbf{2 8}$, R67 (2015).

${ }^{4}$ E. Schöll, "Synchronization patterns and chimera states in complex networks: Interplay of topology and dynamics," Eur. Phys. J. Spec. Top. 225, 891-919 (2016).

${ }^{5}$ O. Omelchenko, "The mathematics behind chimera states," Nonlinearity 31, R121 (2018).

${ }^{6}$ S. Boccaletti, A. N. Pisarchik, C. I. del Genio, and A. Amann, Synchronization: From Coupled Systems to Complex Networks (Cambridge University Press, 2018).

${ }^{7}$ V. A. Maksimenko, V. V. Makarov, B. K. Bera, D. Ghosh, S. K. Dana, M. V. Goremyko, N. S. Frolov, A. A. Koronovskii, and A. E. Hramov, "Excitation and suppression of chimera states by multiplexing," Phys. Rev. E 94, 052205 (2016).

${ }^{8}$ S. Ghosh, A. Kumar, A. Zakharova, and S. Jalan, "Birth and death of chimera: Interplay of delay and multiplexing," Europhys. Lett. 115, 60005 (2016).

${ }^{9}$ S. Majhi, M. Perc, and D. Ghosh, "Chimera states in uncoupled neurons induced by a multilayer structure," Sci. Rep. 6, 39033 (2016).

${ }^{10} \mathrm{~S}$. Majhi, M. Perc, and D. Ghosh, "Chimera states in a multilayer network of coupled and uncoupled neurons," Chaos 27, 073109 (2017).

${ }^{11}$ V. A. Maksimenko, M. V. Goremyko, V. V. Makarov, A. E. Hramov, D. Ghosh, B. K. Bera, and S. K. Dana, "Excitation and suppression of chimeric states in the multilayer network of oscillators with nonlocal coupling," Bull. Russ. Acad. Sci.: Phys. 81, 110-113 (2017). 
${ }^{12}$ R. G. Andrzejak, G. Ruzzene, and I. Malvestio, "Generalized synchronization between chimera states," Chaos 27, 053114 (2017).

${ }^{13}$ M. V. Goremyko, V. A. Maksimenko, V. V. Makarov, D. Ghosh, B. Bera, S. K. Dana, and A. E. Hramov, "Interaction of chimera states in a multilayered network of nonlocally coupled oscillators," Tech. Phys. Lett. 43, 712-715 (2017).

${ }^{14}$ M. V. Goremyko, V. A. Maksimenko, V. V. Makarov, D. Ghosh, B. K. Bera, S. K. Dana, and A. E. Hramov, "Numerical analysis of the chimera states in the multilayered network model," in Dynamics and Fluctuations in Biomedical Photonics XIV (International Society for Optics and Photonics, 2017), Vol. 10063, p. 100631J.

${ }^{15}$ A. Bukh, E. Rybalova, N. Semenova, G. Strelkova, and V. Anishchenko, "New type of chimera and mutual synchronization of spatiotemporal structures in two coupled ensembles of nonlocally interacting chaotic maps," Chaos 27, 111102 (2017)

${ }^{16}$ N. Frolov, A. Koronovskii, V. Makarov, V. Maksimenko, M. Goremyko, and A. Hramov, "Control of pattern formation in complex network by multiplexing," Cybern. Phys. 6, 121-125 (2017).

${ }^{17}$ S. Ghosh, A. Zakharova, and S. Jalan, "Non-identical multiplexing promotes chimera states," Chaos Solitons Fractals 106, 56-60 (2018).

${ }^{18}$ F. Xu, J. Zhang, M. Jin, S. Huang, and T. Fang, "Chimera states and synchronization behavior in multilayer memristive neural networks," Nonlinear Dyn. 1-9 (2018), available at https://link.springer.com/journal/ 11071/onlineFirst/page/8.

${ }^{19}$ Q. Dai, Q. Liu, H. Cheng, H. Li, and J. Yang, "Chimera states in a bipartite network of phase oscillators," Nonlinear Dyn. 92, 741-749 (2018).

${ }^{20}$ N. F. Rulkov, M. M. Sushchik, L. S. Tsimring, and H. D. I. Abarbanel, "Generalized synchronization of chaos in directionally coupled chaotic systems," Phys. Rev. E 51, 980-994 (1995).

${ }^{21}$ H. D. I. Abarbanel, N. F. Rulkov, and M. M. Sushchik, "Generalized synchronization of chaos: The auxiliary system approach," Phys. Rev. E 53, 4528 (1996).

${ }^{22}$ L. Kocarev and U. Parlitz, "Generalized synchronization, predictability, and equivalence of unidirectionally coupled dynamical systems," Phys. Rev. Lett. 76, 1816 (1996).

${ }^{23}$ O. E. Omel'chenko, M. Wolfrum, and Y. L. Maistrenko, "Chimera states as chaotic spatiotemporal patterns," Phys. Rev. E 81, 065201 (2010).

${ }^{24} \mathrm{M}$. Wolfrum and O. E. Omel'chenko, "Chimera states are chaotic transients," Phys. Rev. E 84, 015201 (2011).

${ }^{25}$ M. G. Rosenblum, A. S. Pikovsky, and J. Kurths, "Phase synchronization of chaotic oscillators,” Phys. Rev. Lett. 76, 1804-1807 (1996).

${ }^{26}$ A. Pikovsky, M. Rosenblum, and J. Kurths, Synchronization: A Universal Concept in Nonlinear Sciences (Cambridge University Press, Cambridge, UK, 2001).

${ }^{27}$ Z.-M. Wu, H.-Y. Cheng, Y. Feng, H.-H. Li, Q.-L. Dai, and J.-Z. Yang, "Chimera states in bipartite networks of FitzHugh-Nagumo oscillators," Front. Phys. 13, 130503 (2018).

${ }^{28}$ K. Pyragas, "Conditional Lyapunov exponents from time series," Phys. Rev. E 56, 5183 (1997).

${ }^{29}$ D. Chicharro and R. G. Andrzejak, "Reliable detection of directional couplings using rank statistics," Phys. Rev. E 80, 026217 (2009).

${ }^{30}$ Y. Kuramoto, Chemical Oscillations, Waves, and Turbulence (Springer, 1984).
${ }^{31}$ References 47 and 48 used a similar coupling from a mean field to the phases of individual oscillators between two populations of oscillators. In contrast to these studies, we do not use the amplitude of the mean field as factor of the coupling term.

${ }^{32}$ R. G. Andrzejak, C. Rummel, F. Mormann, and K. Schindler, "All together now: Analogies between chimera state collapses and epileptic seizures," Sci. Rep. 6, 23000 (2016).

${ }^{33}$ Unless stated otherwise, we unwrap the order parameter phases $\Phi_{x}(t)$ and $\Phi_{y}(t)$ from the circular domain $(0,2 \pi]$ to the infinite domain. For example, 1.5 rotations correspond to $\pi$ in the circular domain but to $3 \pi$ in the infinite domain.

${ }^{34}$ In more strict terms, for uncoupled layers $C$ converges to zero from above for increasing length of the evaluation interval $I$.

${ }^{35}$ M. H. DeGroot and M. J. Schervish, Probability and Statistics (Pearson Education, 2012).

${ }^{36}$ J. Lehnert, T. Dahms, P. Hövel, and E. Schöll, "Loss of synchronization in complex neuronal networks with delay," Europhys. Lett. 96, 60013 (2011).

${ }^{37}$ A. Avena-Koenigsberger, B. Misic, and O. Sporns, "Communication dynamics in complex brain networks," Nat. Rev. Neurosci. 19, 17 (2018).

${ }^{38}$ G. Buzsáki and A. Draguhn, "Neuronal oscillations in cortical networks," Science 304, 1926-1929 (2004).

${ }^{39}$ P. J. Uhlhaas and W. Singer, "Neural synchrony in brain disorders: Relevance for cognitive dysfunctions and pathophysiology," Neuron 52, 155-168 (2006).

${ }^{40}$ F. Fröhlich and D. A. McCormick, "Endogenous electric fields may guide neocortical network activity," Neuron 67, 129-143 (2010).

${ }^{41}$ E. H. Toloza, E. Negahbani, and F. Fröhlich, " $I_{\mathrm{h}}$ interacts with somatodendritic structure to determine frequency response to weak alternating electric field stimulation," J. Neurophysiol. 119, 1029-1036 (2017).

${ }^{42}$ F. Aspart, M. W. Remme, and K. Obermayer, "Differential polarization of cortical pyramidal neuron dendrites through weak extracellular fields," PLoS Comput. Biol. 14, e1006124 (2018).

${ }^{43}$ P. Jiruska, M. De Curtis, J. G. Jefferys, C. A. Schevon, S. J. Schiff, and K. Schindler, "Synchronization and desynchronization in epilepsy: Controversies and hypotheses," J. Physiol. 591, 787-797 (2013).

${ }^{44}$ V. Gnatkovsky, M. De Curtis, C. Pastori, F. Cardinale, G. Lo Russo, R. Mai, L. Nobili, I. Sartori, L. Tassi, and S. Francione, "Biomarkers of epileptogenic zone defined by quantified stereo-EEG analysis," Epilepsia 55, 296-305 (2014).

${ }^{45}$ K. Schindler, H. Leung, C. E. Elger, and K. Lehnertz, "Assessing seizure dynamics by analysing the correlation structure of multichannel intracranial EEG," Brain 130, 65-77 (2006).

${ }^{46}$ M. R. Bower, M. Stead, F. B. Meyer, W. R. Marsh, and G. A. Worrell, "Spatiotemporal neuronal correlates of seizure generation in focal epilepsy," Epilepsia 53, 807-816 (2012).

${ }^{47} \mathrm{~K}$. Okuda and Y. Kuramoto, "Mutual entrainment between populations of coupled oscillators," Prog. Theor. Phys. 86, 1159-1176 (1991).

${ }^{48}$ E. Montbrió, J. Kurths, and B. Blasius, "Synchronization of two interacting populations of oscillators," Phys. Rev. E 70, 056125 (2004). 